\title{
Spitzer observations of a circumstellar nebula around the candidate luminous blue variable MWC 930
}

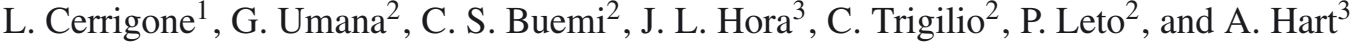 \\ ${ }^{1}$ Centro de Astrobiología, INTA-CSIC ctra de Ajalvir km 4, 28850 Torrejón de Ardoz, Spain \\ e-mail: cerrigone@cab.inta-csic.es \\ 2 INAF-Osservatorio Astrofisico di Catania, via S. Sofia 78, 95123 Catania, Italy \\ ${ }^{3}$ Harvard-Smithsonian Center for Astrophysics, 38 Garden St., Cambridge MA 02138, USA
}

Received 21 November 2013 / Accepted 21 December 2013

\begin{abstract}
Context. The source MWC 930 is a star just $\sim 2^{\circ}$ above the Galactic plane whose nature is not clear and has not been studied in detail so far. While a post-asymptotic giant branch classification was proposed in the past, studies of its optical spectrum and photometry pointed toward strong variability, therefore, the object was reclassified as a luminous blue variable (LBV) candidate.

Aims. The LBVs typically undergo phases of strong mass loss in the form of eruptions that can create shells of ejecta around the star. Our goal is to search for the presence of such a circumstellar nebula in MWC 930 and investigate its properties.

Methods. To do so, we make use of space-based infrared data from our Spitzer campaign performed with the InfraRed Array Camera (IRAC) and the InfraRed Spectrograph (IRS), as well as data from optical and infrared (IR) surveys.

Results. In our Spitzer images, we clearly detect an extended shell around MWC 930 at wavelengths longer than $5 \mu \mathrm{m}$. The midinfrared spectrum is dominated by the central star and mostly shows forbidden lines of [FeII] with an underlying continuum that decreases with wavelengths up to $\sim 15 \mu \mathrm{m}$ and then inverts its slope, displaying a second peak around $60 \mu \mathrm{m}$, which is evidence of cold dust grains formed in a past eruption. By modeling the spectral energy distribution, we identify two central components, beside the star and the outer shell. These extra sources of radiation are interpreted as material close to the central star, which may be due to a recent ejection. Features of C-bearing molecules or grains are not detected.
\end{abstract}

Key words. circumstellar matter - stars: mass-loss - stars: winds, outflows - stars: individual: MWC 930 - infrared: stars

\section{Introduction}

Luminous blue variables (LBVs) are rare massive (M ZAMS $\gtrsim$ $\left.25 M_{\odot}\right)$ stars with high luminosities $\left(L \geq 10^{5.4} L_{\odot}\right)$ subject to variability. Two groups of LBVs are identified. The first one includes those stars that display spectral and photometric variability similar to that of S Dor, the classical LBV prototype. These sources are subject to episodic outbursts with variations of 1-2 mag at approximately a constant bolometric luminosity over a time of years or decades. The second group includes stars characterized by giant eruptions, when the visual brightness of the star grows on an order of magnitude and the bolometric luminosity is also increased (Humphreys \& Davidson 1994). The latter group contains only P Cyg and $\eta$ Car in the Galaxy.

The origin of the instability of these stars is not clear, but it is commonly accepted that it must be related to them being close to the Eddington limit for stability against radiation pressure (Nota et al. 1995). The limit is expressed as the dimensionless $\Gamma$ parameter, defined as the ratio of radiative to gravitational acceleration, which is proportional to the ratio of stellar luminosity to mass $\left(\Gamma_{\text {Edd }} \propto L / M\right)$. The less luminous members of the group can be as close to the Eddington limit as the more luminous members if they have undergone a heavy mass loss during a previous red supergiant phase.

From an evolutionary point of view, LBVs are considered as transition objects between $\mathrm{O}$ stars and Wolf-Rayet (WR) stars, which eventually explode as supernovae (SN). However, this evolutionary path has been recently challenged by observations that indicate how (perhaps only some) LBVs may directly go into the SN phase (Vink 2012).

Our knowledge of the LBV phenomenon is hampered by the paucity of stars that display it. So far, only 14 such stars are confirmed LBVs in our Galaxy (Vink 2012). To be confirmed as an LBV, a star must at least show both the spectral-type and photometric variability observed in S Dor-type sources, keeping in mind that this phenomenon may be intermittent in a star's life. The presence of a circumstellar nebula around a supergiant is evidence of past high mass loss by the star and can be taken as an indication that the star is an LBV candidate.

\subsection{MWC 930}

Optical spectra of MWC 930 display Balmer lines in emission, as well as permitted and forbidden emission lines of [FeII] (Parthasarathy et al. 2000). Using low-resolution spectra, Gauba et al. (2003) detected $\mathrm{H} \alpha$ in emission and a weak circumstellar nebulosity of less than $2^{\prime \prime}$. They concluded that the target may be a hot post asymptotic giant branch (AGB) star at a distance of about 200 pc.

Miroshnichenko et al. (2005) performed long-term photometric monitoring of the source and acquired high-resolution optical spectra. In their data, they found strong photometric variability (inappropriate of a post-AGB star) and several spectral lines with $\mathrm{P}$ Cyg profiles, pointing to a terminal wind velocity of $\sim 140 \mathrm{~km} \mathrm{~s}^{-1}$. They also estimated a lower limit to the massloss rate of $1.5 \times 10^{-6} M_{\odot} \mathrm{yr}^{-1}$, which also does not match the 
Table 1. Optical and infrared photometric data for MWC 930 (star + nebula).

\begin{tabular}{lccccc}
\hline \hline Optical & $B$ & $V$ & $R$ & & \\
$\mathrm{~S}_{v}(\mathrm{Jy})$ & 0.006 & 0.028 & 0.156 & & \\
\hline $2 \mathrm{MASS}$ & $J$ & $H$ & $K \mathrm{~s}$ & & \\
$\mathrm{~S}_{v}(\mathrm{Jy})$ & 3.48 & 4.86 & 5.24 & & \\
\hline IRAC & $3.6 \mu \mathrm{m}$ & $4.5 \mu \mathrm{m}$ & $5.8 \mu \mathrm{m}$ & $8.0 \mu \mathrm{m}$ & \\
$\mathrm{S}_{v}(\mathrm{Jy})$ & $8.5 \pm 0.3$ & $7.5 \pm 0.2$ & $6.1 \pm 0.2$ & $5.14 \pm 0.15$ & \\
\hline WISE & $3.4 \mu \mathrm{m}$ & $4.6 \mu \mathrm{m}$ & $12 \mu \mathrm{m}$ & $22 \mu \mathrm{m}$ & \\
$\mathrm{S}_{v}($ Jy $)$ & $8.6 \pm 0.9$ & $13 \pm 1$ & $1.6 \pm 0.2$ & $4.3 \pm 0.4$ & \\
\hline AKARI & $9 \mu \mathrm{m}$ & $65 \mu \mathrm{m}$ & $90 \mu \mathrm{m}$ & $140 \mu \mathrm{m}$ & $160 \mu \mathrm{m}$ \\
$\mathrm{S}_{v}(\mathrm{Jy})$ & $3.25 \pm 0.02$ & $15 \pm 1$ & $12.5 \pm 0.8$ & $8 \pm 1$ & $4.38^{a}$ \\
\hline IRAS & $12 \mu \mathrm{m}$ & $25 \mu \mathrm{m}$ & $60 \mu \mathrm{m}$ & $100 \mu \mathrm{m}$ & \\
$\mathrm{S}_{v}(\mathrm{Jy})$ & $1.8 \pm 0.2$ & $4.0 \pm 0.3$ & $38 \pm 4$ & $35 \pm 3$ & \\
\hline
\end{tabular}

Notes. ${ }^{(a)}$ No error listed in the catalog.

spectral characteristics of a star in the post-AGB evolutionary stage. Their spectral analysis indicates a central star temperature of $22000 \pm 5000 \mathrm{~K}$ and a luminosity of $10^{5.5 \pm 0.2} L_{\odot}$, with their estimate of the distance to MWC 930 of $3.5 \mathrm{kpc}$. These values rule out a post-AGB classification and make the star a candidate LBV.

Subsequent studies confirmed the spectral classification as a B5-B9 star with emission lines that show P Cyg profiles (Carmona et al. 2010) and Brackett emission lines in the nearIR without detectable CO bands (Venkata Raman \& Anandarao 2008).

\section{Observations and results}

We observed MWC 930 with the Infrared Array Camera (IRAC; Fazio et al. 2004) and the Infrared Spectrograph (IRS; Houck et al. 2004) on board the Spitzer space telescope (Werner et al. 2004) within program 50116 (PI: G. Fazio). The IRAC images were obtained on 2009 May 01 (AOR 25444608) and the IRS spectra two days later (AOR 25444352). For both instruments, the pointing coordinates were 18:26:25.24 in right ascension and -07:13:17.7 in declination (J2000).

\subsection{Spitzer IRAC observations}

The infrared imaging was performed at 3.6, 4.5, 5.8, and $8.0 \mu \mathrm{m}$ in high dynamic range mode. For each target, we obtained eight dithered frames for a total on-source time of about $96 \mathrm{~s}$ per IRAC channel. Corrected basic calibrated data (cBCDs) were retrieved from the Spitzer archive (pipeline version S18.25.0) and then coadded with a sampling of $0.3^{\prime \prime}$ per pixel using MOPEX (Makovoz et al. 2006). These are BCDs, where artefact mitigation software has been applied. Photometry on the central source was performed by point-spread function (PSF) subtraction in the final mosaics with IRACproc (Schuster et al. 2006).

Since the central star is bright, we extracted our photometry using PSFs that were specifically developed to perform photometry even on heavily saturated objects. The PSFs had been obtained from observations of bright stars and then directly normalized to the IRAC observations of Vega (Marengo et al. 2006, 2007). Therefore, by fitting the low-level features (diffraction spikes and wings) of the saturated PSFs of the observed source, magnitudes can be determined with an accuracy better than $3 \%$, which is independent of the standard IRAC flux calibration (Schuster et al. 2006).
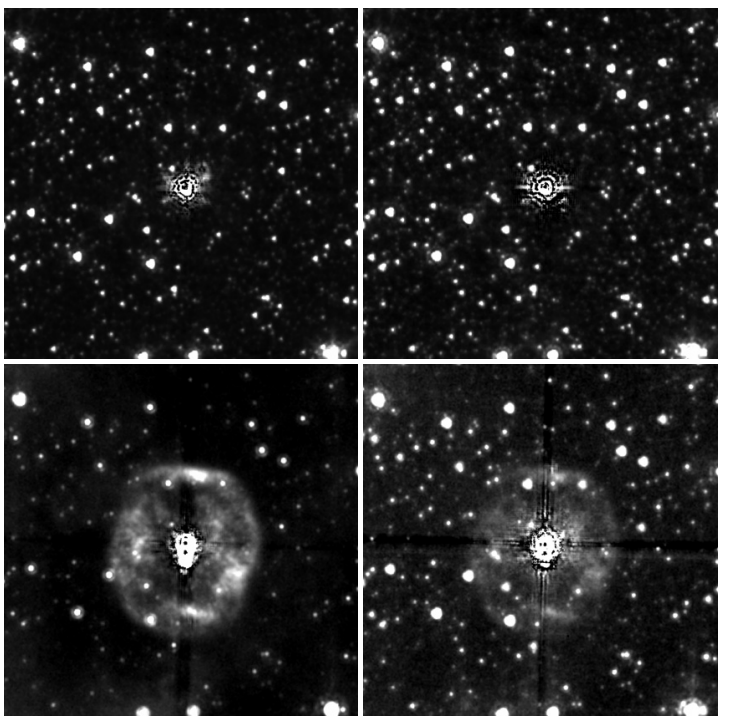

Fig. 1. IRAC images after PSF subtraction. Clockwise from top left: 3.6, $4.5,5.8$, and $8.0 \mu \mathrm{m}$. Each image is about $200^{\prime \prime}$ wide and aligned with north up and east to the left.

We thus obtained the photometry of the central source in all of the four IRAC channels. In the 5.8 and $8.0 \mu \mathrm{m}$ bands, we clearly detect an extended detached shell beyond the central star, which is brighter at 8.0 than at $5.8 \mu \mathrm{m}$ (Fig. 1). The size of the nebula is about $100^{\prime \prime} \times 85^{\prime \prime}$. At the assumed distance of $3.5 \mathrm{kpc}$, this implies a physical size of about $1.70 \mathrm{pc} \times 1.44 \mathrm{pc}$. The nebula has an elliptical shape with clumps in both the north-south and (roughly) east-west directions.

To better investigate the nature of this detached shell, we searched the web archive of the Wide-field Infrared Survey Explorer (WISE; Wright et al. 2010) and retrieved its images of MWC 930 in all bands. While in the first two shorter-wavelength bands the emission is primarily point-like, at 12 and $22 \mu \mathrm{m}$ the detached shell seen with IRAC is detected, as can be seen in Fig. 2. In these two bands, we performed aperture photometry, since the values listed in the WISE catalog (obtained by PSF fitting) do not include the emission over the whole outer nebula. Our IRAC and WISE photometric data are reported in Table 1, where the flux densities are referred to the whole emitting source (central star + detached nebula). 

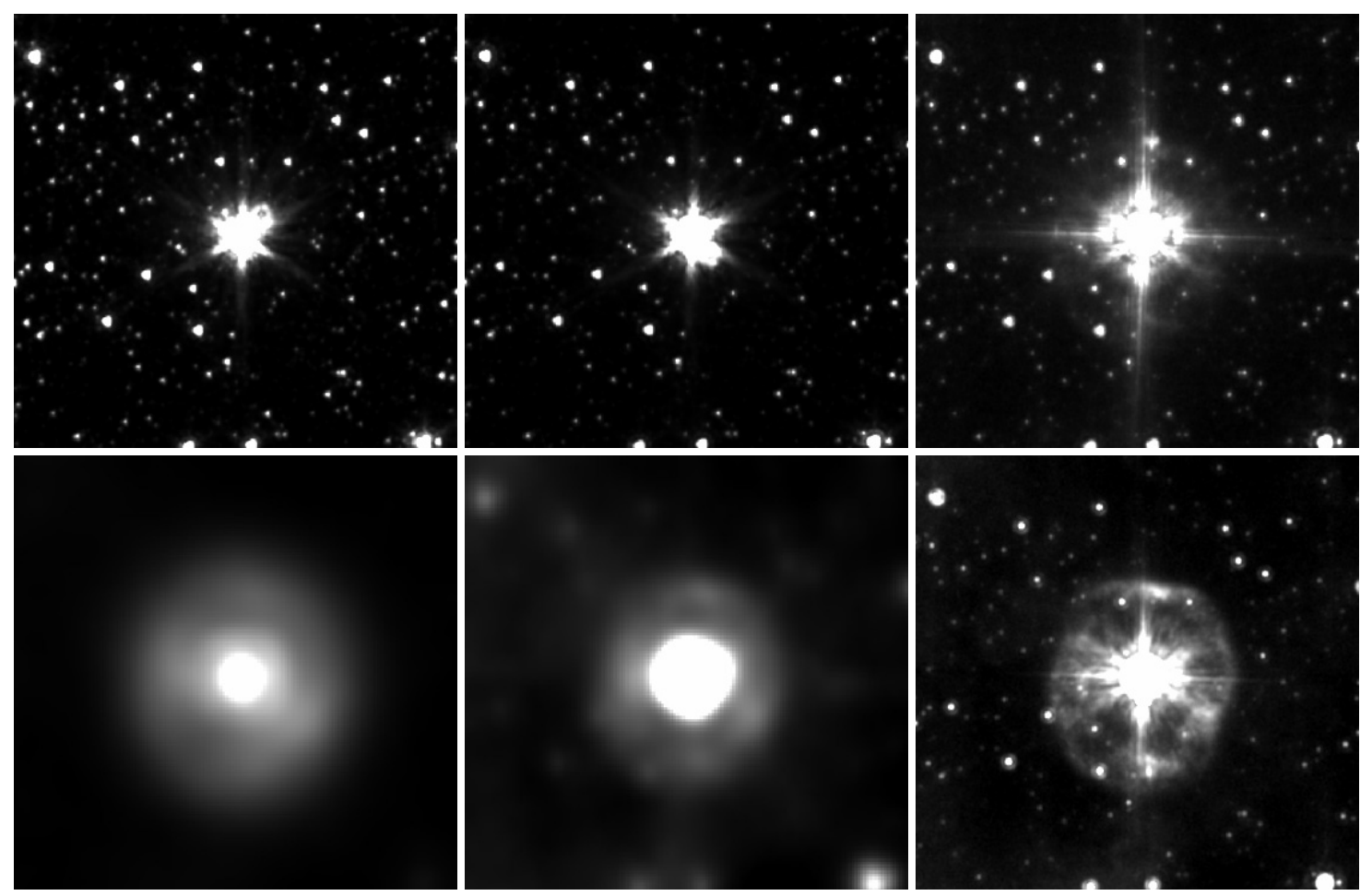

Fig. 2. IRAC and WISE images of MWC 930. Clockwise from top left: The IRAC images at 3.6, 4.5, 5.8, and 8.0 $\mu$ m and then the WISE images at 12 and $22 \mu \mathrm{m}$. Size and orientation as in Fig. 1.

Table 2. IRAC flux densities of the two components in MWC 930.

\begin{tabular}{lcccc}
\hline \hline MWC 930 & $3.6 \mu \mathrm{m}$ & $4.5 \mu \mathrm{m}$ & $5.8 \mu \mathrm{m}$ & $8.0 \mu \mathrm{m}$ \\
& $\mathrm{Jy}$ & $\mathrm{Jy}$ & $\mathrm{Jy}$ & $\mathrm{Jy}$ \\
\hline Central source & $8.5 \pm 0.3$ & $7.5 \pm 0.2$ & $5.2 \pm 0.2$ & $4.0 \pm 0.1$ \\
Nebula & - & - & $0.89 \pm 0.09$ & $1.14 \pm 0.1$ \\
\hline
\end{tabular}

We also performed aperture photometry on the 5.8 and $8.0 \mu \mathrm{m}$ IRAC images after PSF subtraction and could, thus, calculate the flux density of the detached nebula, as reported in Table 2.

\subsection{Spitzer IRS observations}

Observations with the IRS were carried out at low spectral resolution $(R \sim 60-120)$. We used both the short-low (SL) and the long-low (LL) IRS modules. Each module had two orders, which covered the 5.2-7.7 $\mu \mathrm{m}$ and 7.4-14 $\mu \mathrm{m}$ ranges at short wavelengths (SL module) and the 14.0-21.3 $\mu \mathrm{m}$ and 19.5-38.0 $\mu \mathrm{m}$ ranges at long wavelengths (LL module). The target was observed with a ramp time of $6 \mathrm{~s}$ per cycle and two cycles per module were performed. We retrieved the IRS BCDs (pipeline version S18.18.0) from the Spitzer archive. At low resolution the target is observed in two different slit positions; background subtraction was performed by using the observation at one nod position as a background for the other. The spectra were extracted with SMART ${ }^{1}$ (Higdon et al. 2004) with the optimal extraction algorithm. The IRS slits are overlayed in Fig. 3 on the IRAC $8.0 \mu \mathrm{m}$ image of MWC 930.

1 SMART was developed by the IRS Team at Cornell University and is available through the Spitzer Science Center at Caltech.
We also extracted the spectra from BCDs, where we had subtracted the background in a different way. Each IRS lowresolution module had two orders observing at the same time but not in the same direction; hence, when one was on target, the other would perform a sky observation. We combined these offtarget observations together and subtracted them from the corresponding on-target $\mathrm{BCDs}$. The main difference between the two methods of background subtraction is that while the second can actually show contributions from a circumstellar nebula, the first (nod subtraction) eliminates any contribution to the spectrum from possible circumstellar nebulosity (assuming this is homogeneous within the slit).

For the two different background subtractions, we also extracted the spectra with both the optimal algorithm and the full slit (appropriate for extended sources). No differences were found between the optimal and full-slit spectra, which indicates that the fraction of flux collected from the detached nebula by the IRS modules is likely negligible. A small difference was instead observed between the spectra extracted from BCDs with different background subtraction.

As can be seen in Fig. 4, both the spectrum from the nodsubtracted BCDs and that from the BCDs obtained with the offtarget subtraction show an underlying continuum excess at long wavelengths. This excess appears to be approximately constant with wavelength in the nod-subtracted and increases with wavelength beyond $\sim 23 \mu \mathrm{m}$ in the off-subtracted data. As mentioned above, this means that a small fraction of emission from the circumstellar nebula is caught by the detectors and more efficiently eliminated by the nod subtraction. Below $\sim 23 \mu \mathrm{m}$, the emission within the slit seems dominated by the central source, as shown by the spectrum, that is reasonably matched by the IRAC photometric data of the central source alone. 


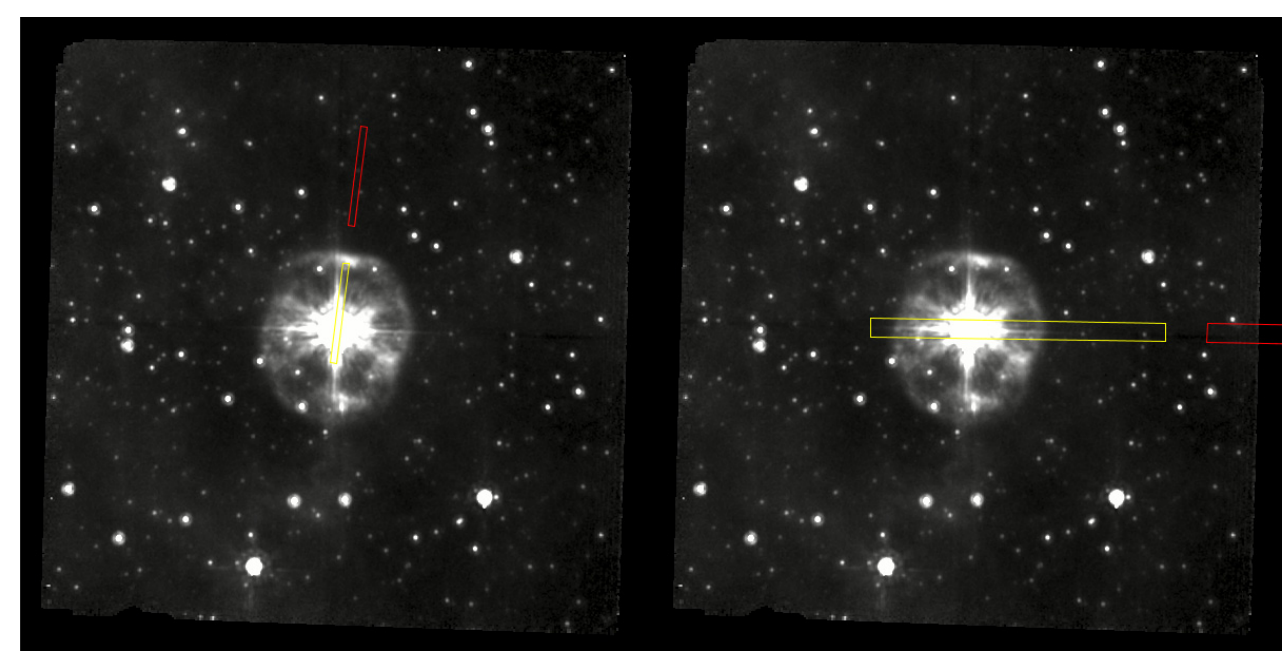

Fig. 3. Overlay of the IRS slits on the IRAC image at $8 \mu \mathrm{m}$ : on the left side the two orders of the SL module and on the right side those of the LL one.

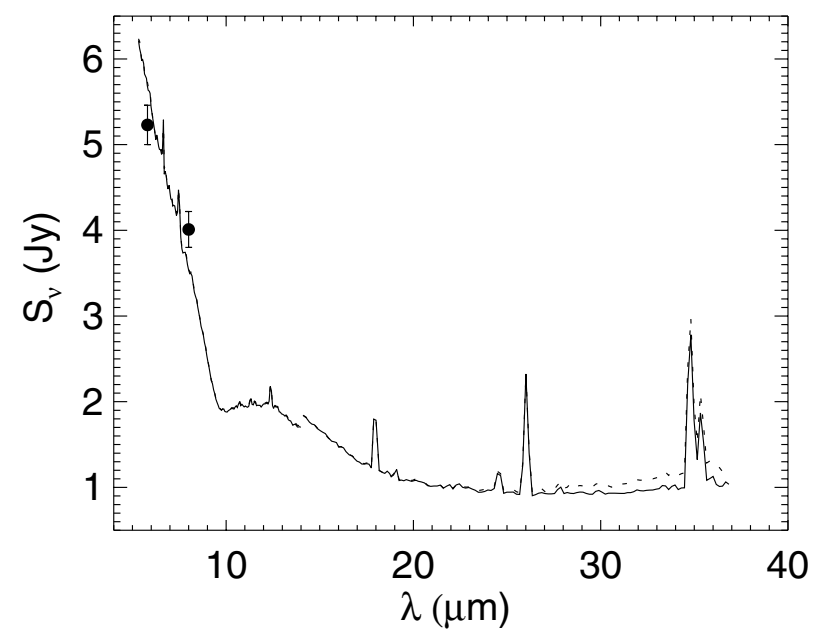

Fig. 4. IRS spectrum of MWC 930 extracted from nod-subtracted (solid line) and off-subtracted (dotted line) BCDs. IRAC photometric measurements at 5.8 and $8.0 \mu \mathrm{m}$ are also displayed.

The continuum excess at long wavelengths can be interpreted as due to thermal emission from circumstellar dust. Since only a small fraction of the circumstellar nebula falls within the IRS slit (Fig. 3), it is not possible to rely on the IRS data to derive the temperature of the emitting dust.

Finally, we extracted the spectrum from the off-subtracted BCDs at positions that are spaced $2^{\prime \prime}$ from one another along the slit. This was done to check whether the nebular emission could be detected by moving away from the bright central star. However, our inspection of the spectra did not reveal any detectable nebular radiation.

\section{The IRS spectrum: atomic lines}

Several lines can be identified in the IRS spectrum of MWC 930. Almost all are due to ionized elements and, in particular, to Fe II. For some lines, the low spectral resolution of our data does not allow us to clearly attribute the feature to one element. In particular, almost all the lines of He II and H I are too close to be distinguished from one another. We notice that HeI lines were detected in previous studies of LBVs performed with the IRS at higher spectral resolution, but not [He II] (Umana et al. 2009, 2010). Furthermore, the He II lines would imply a high level of nebular excitation, which is not supported by the detection of highly excited lines of other species. A summary of the lines detected is given in Table 3 .

The inspection of the IRS data and, especially, the extraction of the spectrum at different positions along the slit indicate that the lines do not originate in the extended nebula. Instead, they are found to arise from the central region.

Among the lines detected, that of [FeII] at $26 \mu \mathrm{m}$ and of [SiII] at $34.8 \mu \mathrm{m}$ can be compared to the model plots derived by Kaufman et al. (1999) and Kaufman et al. (2006) in photo dissociation regions (PDRs). The average radiation field acting on the PDR around the star can be described by the parameter $G$, which is the intensity of the UV radiation field in units of the average interstellar radiation field flux in the far ultraviolet range (from $6 \mathrm{eV}$ to $13.6 \mathrm{eV}$ ) (Habing 1968):

$G=\frac{L F_{\mathrm{UV}}}{4 \pi R_{i}^{2} G_{0}}$

The parameter $G$ depends on the stellar luminosity $L$, the fraction of energy $F_{\mathrm{UV}}$ at wavelengths shorter than $6 \mathrm{eV}(\sim 2066 \AA)$ in a blackbody spectrum at the same temperature as the star, the inner radius $R_{i}$ from the star to the PDR and the average UV flux $G_{0}$ in the diffuse ISM $\left(G_{0}=1.6 \times 10^{-3} \mathrm{erg} \mathrm{cm}^{-2} \mathrm{~s}^{-1}\right.$; Draine 1978).

Since we have noticed that the lines arise in a region close to the star and not in the outer nebula, we do not have a direct estimate of $R_{i}$. The two IRS slits have widths of $\sim 4^{\prime \prime}$ (SL) and $\sim 10^{\prime \prime}$ (LL). If we take half of the LL slit width as an upper limit to the inner radius scaled to the assumed distance of $3.5 \mathrm{kpc}$ $\left(R_{i} \leq 2.62 \times 10^{17} \mathrm{~cm}\right)$, we find $G \sim 6.7 \times 10^{5} G_{0}$. Gauba et al. (2003) detected a possible nebulosity around the central star and gave $2^{\prime \prime}$ as an upper limit to its radius $\left(R_{i} \leq 1.05 \times 10^{17} \mathrm{~cm}\right)$. Such a value for $R_{i}$ would give us $G \sim 4.2 \times 10^{6} G_{0}$.

To estimate the density in the PDR, we make use of the plots given by Kaufman et al. (2006) for the intensity of the [SiII] and [FeII] lines as a function of $n_{e}$ and $G$, which we display in Fig. 5. The model used to obtain the plots had the following elemental abundances: $[\mathrm{C}] /[\mathrm{H}]=1.4 \times 10^{-4},[\mathrm{O}] /[\mathrm{H}]=3.2 \times$ $10^{-4},[\mathrm{Si}] /[\mathrm{H}]=1.7 \times 10^{-6},[\mathrm{~S}] /[\mathrm{H}]=2.8 \times 10^{-5},[\mathrm{Fe}] /[\mathrm{H}]=$ $1.7 \times 10^{-7},[\mathrm{Mg}] /[\mathrm{H}]=1.1 \times 10^{-6}$, and $[\mathrm{PAH}] /[\mathrm{H}]=6 \times 10^{-7}$.

If we take $5^{\prime \prime}$ as the radius of the emitting region, we can calculate from the fluxes in Table 3 the following intensities for the [SiII] and [FeII] lines, respectively: $\sim 9.21 \times 10^{-4}$ and $\sim 9.05 \times 10^{-4} \mathrm{erg} \mathrm{cm}^{-2} \mathrm{~s}^{-1} \mathrm{sr}^{-1}\left(5.8 \times 10^{-3}\right.$ and $5.6 \times$ $10^{-3} \mathrm{erg} \mathrm{cm}^{-2} \mathrm{~s}^{-1} \mathrm{sr}^{-1}$ for an inner radius of $2^{\prime \prime}$, respectively). By combining these values with that of $G$, we can try to estimate 
Table 3. Atomic lines detected in the IRS spectrum.

\begin{tabular}{lcccc}
\hline \hline Species & $\begin{array}{c}\text { Exp. } \lambda_{\mathrm{c}} \\
\mu \mathrm{m}\end{array}$ & $\begin{array}{c}\text { Obs. } \lambda_{\mathrm{c}} \\
\mu \mathrm{m}\end{array}$ & $\begin{array}{c}F W H M \\
10^{-3} \mu \mathrm{m}\end{array}$ & $\begin{array}{c}\text { Flux } \\
10^{-13} \mathrm{erg} \mathrm{cm}^{-2} \mathrm{~s}^{-1}\end{array}$ \\
\hline$[\mathrm{NiII}]$ & 6.6360 & $6.64 \pm 0.01$ & $63 \pm 7$ & $30 \pm 11$ \\
$\mathrm{HeII} / \mathrm{HI}$ & 7.45682 & $7.47 \pm 0.01$ & $106 \pm 3$ & $31 \pm 3$ \\
{$[\mathrm{NiII}]$} & 10.6822 & $10.70 \pm 0.01$ & $119 \pm 3$ & $2.3 \pm 0.2$ \\
{$[\mathrm{NiI}]$} & 11.3075 & $11.31 \pm 0.01$ & $87 \pm 4$ & $2.0 \pm 0.3$ \\
$\mathrm{HeII} / \mathrm{HI}$ & 12.3669 & $12.37 \pm 0.01$ & $118 \pm 4$ & $6.8 \pm 0.7$ \\
{$[\mathrm{FeII}]$} & 17.9359 & $17.94 \pm 0.01$ & $108 \pm 3$ & $16 \pm 2$ \\
$\mathrm{HI}$ & 18.6152 & $18.61 \pm 0.02$ & $179 \pm 6$ & $0.67 \pm 0.08$ \\
$\mathrm{HeII} / \mathrm{HI}$ & 19.0498 & $19.04 \pm 0.01$ & $114 \pm 11$ & $3 \pm 2$ \\
{$[\mathrm{FeII}]$} & 24.5193 & $24.55 \pm 0.02$ & $296 \pm 22$ & $4 \pm 1$ \\
{$[\mathrm{FeII}]$} & 25.9883 & $26.02 \pm 0.01$ & $254 \pm 2$ & $16.7 \pm 0.3$ \\
{$[\mathrm{HeII}]$} & 27.8255 & $27.81 \pm 0.03$ & $365 \pm 26$ & $1.6 \pm 0.4$ \\
{$[\mathrm{SiII}]$} & 34.8152 & $34.79 \pm 0.02$ & $338 \pm 18$ & $17 \pm 3$ \\
{$[\mathrm{FeII}]$} & 35.3487 & $35.36 \pm 0.01$ & $308 \pm 12$ & $7.2 \pm 0.9$ \\
\hline
\end{tabular}
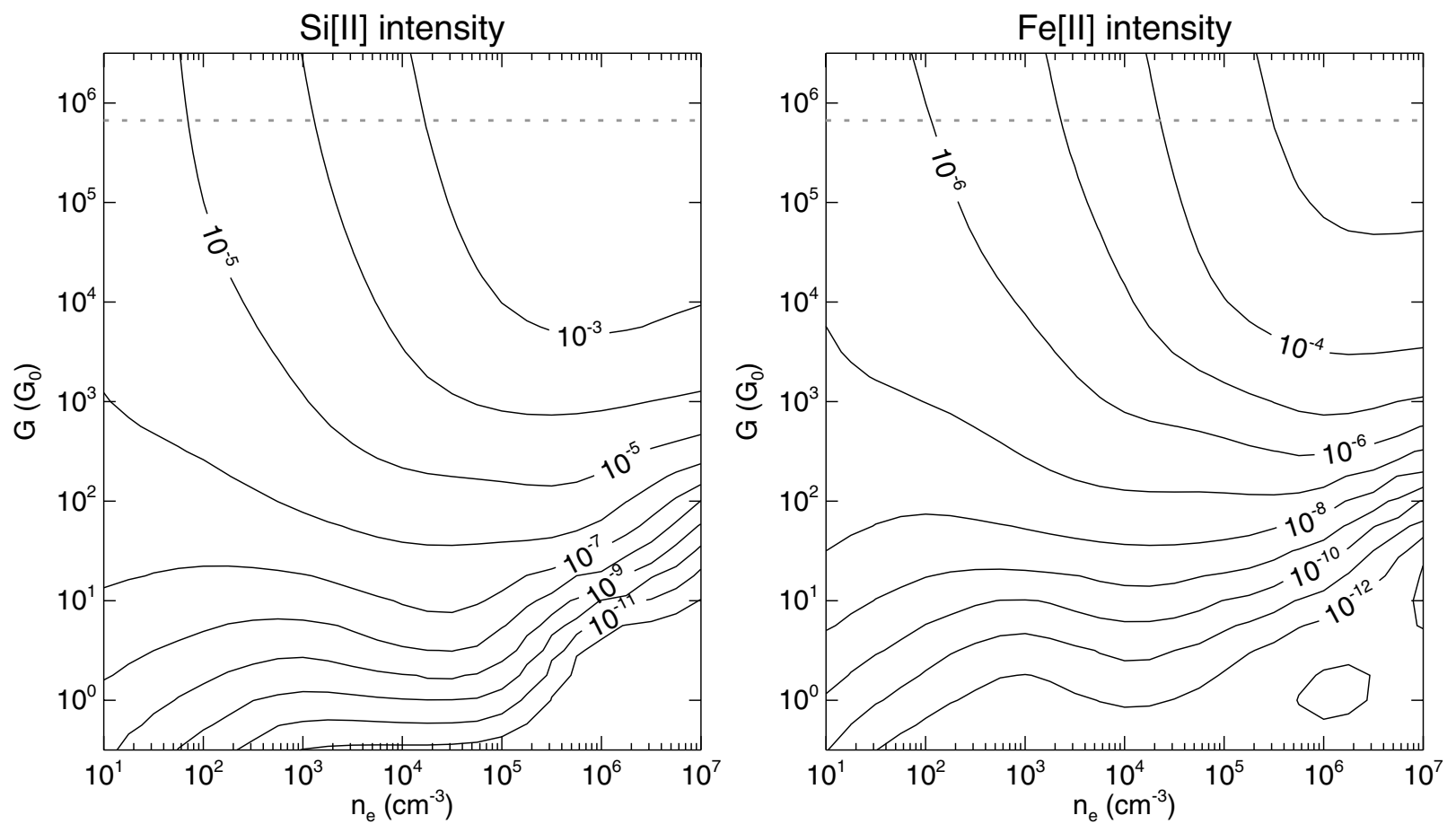

Fig. 5. Plots of the [SiII] (left) and [FeII] (right) line intensities in $\mathrm{erg}^{-2} \mathrm{~cm}^{-1} \mathrm{sr}^{-1}$ as a function of density and local radiation field from the PDR models by Kaufman et al. (2006). The dotted line indicates $G=6.7 \times 10^{5} G_{0}$.

the density of the PDR from the model plots. The [SiII] line intensity implies a density of $\sim 2 \times 10^{4} \mathrm{~cm}^{-3}$, while the [FeII] line gives a value of $\sim 3 \times 10^{5} \mathrm{~cm}^{-3}$, which is about one order of magnitude larger. Densities of the order of $10^{6} \mathrm{~cm}^{-3}$ are reached instead if the inner radius is set to $2^{\prime \prime}$.

Two main factors affect the discrepancy between the density values derived from the different lines: the uncertainty in the value of the inner radius of the PDR and the assumption that both lines are excited at the same distance. Assuming $5^{\prime \prime}$ is an upper limit for the radius of the PDR, we conclude that the emitting region has a high density at least of the order of $10^{4} \mathrm{~cm}^{-3}$.

\section{The IRS spectrum: solid-state spectral features}

Unlike other LBVs, where Spitzer spectra display features of large C-bearing molecules, such as polycyclic aromatic hydrocarbons (PAHs; Umana et al. 2009, 2010), MWC 930 does not display signs of these or other carbonaceous components.
One of the clear features detected in the spectrum of MWC 930 is a deep absorption around $10 \mu \mathrm{m}$. A well-known broad feature centered around $9.7 \mu \mathrm{m}$ is typical of amorphous silicate grains, which is typically seen in absorption against a bright continuum (like in the Galactic center) or in emission (or self-absorption) in O-rich AGB/post-AGB stars and red supergiants (Molster et al. 2010).

To illustrate the shape of this feature in our target and more easily compare it to similar features in other sources, we have fitted a local continuum (linear in the log-log plane) and then derived the optical depth of the feature by considering $F_{\text {obs }}(\lambda)=$ $F_{\text {cont }}(\lambda) \mathrm{e}^{-\tau(\lambda)}$. Figure 6 displays the fitted continuum and the resulting optical depth curve. The profile is compared to that given by Chiar \& Tielens (2006) for the silicate feature in the Galactic center (GC), obtained by the observation of GCS 3 in the Quintuplet Cluster.

In general, the peak wavelength and shape of the feature is due to the exact mixture of silicates (olivines versus pyroxenes, 

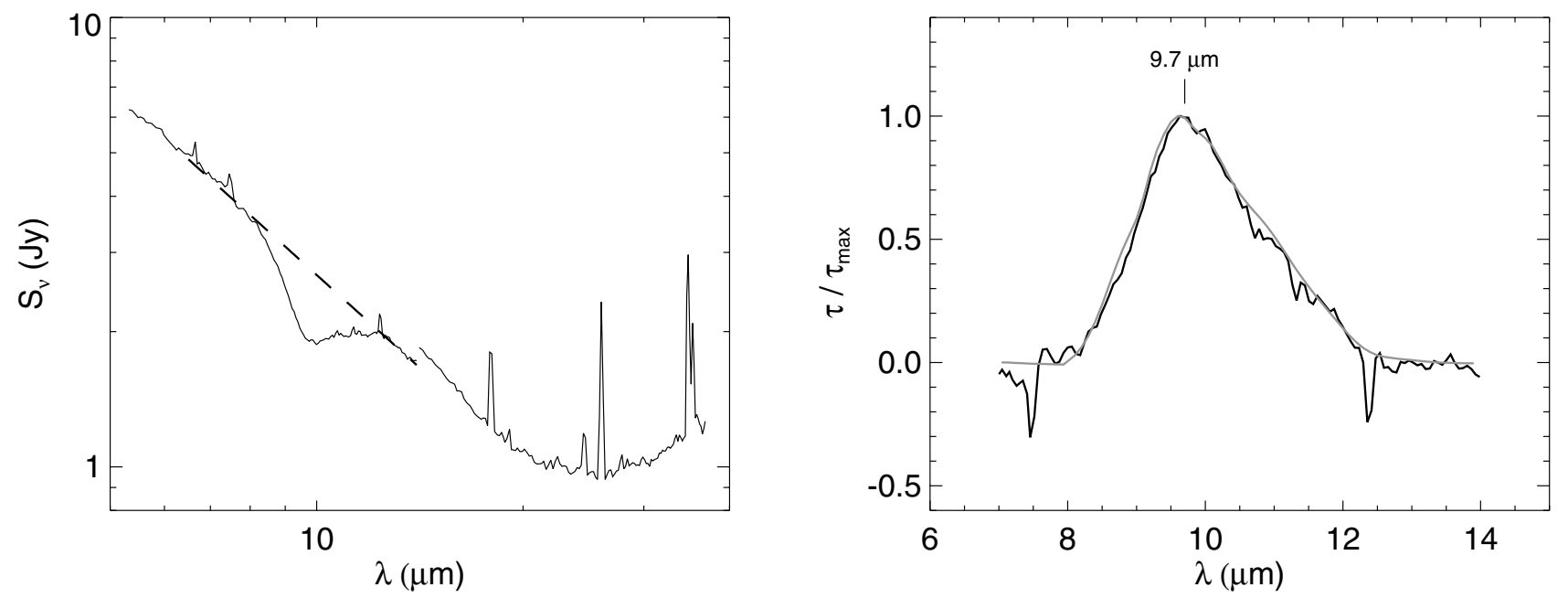

Fig. 6. Left: silicate feature observed in absorption in the spectrum of MWC 930 with the fitted local continuum represented as a dashed line. Right: optical-depth profile of the same feature (black line) compared to that observed toward the Galactic center (gray line) by Chiar \& Tielens (2006).

for example) and size of the grains. The presence of crystalline silicates typically makes the shape change from a peak into a more structured and flatter profile. In MWC 930, we see a relatively smooth profile that overlaps very well with that seen in absorption toward the GC, therefore, we can conclude that it is mostly due to amorphous grains.

At the same time, the profile in MWC 930 does not differ much from that observed in AGB stars with moderate mass-loss rates $\left(\sim 10^{-6} \dot{M}_{\odot} \mathrm{yr}^{-1}\right)$, such as Mira (Molster et al. 2010). In these stars, however, the feature is typically seen in emission from a hot (several $100 \mathrm{~K}$ ) circumstellar dust layer.

The similarity of the silicate profile in MWC 930 with that of the same feature, as seen toward the GC (which is due to interstellar dust), casts some doubt on the circumstellar origin of the feature in our target. The Galactic coordinates of MWC 930 are $l \sim 23.65$ and $b \sim 2.23$; it is therefore relatively close to the Galactic plane. In the IRAS Atlas at 60 and $100 \mu \mathrm{m}$, we can see diffuse emission from cold gas and/or dust around our target (Fig. 7). We further investigate the nature of this feature in Sect. 5, when modeling the spectral energy distribution (SED).

\section{Spectral energy distribution}

To further investigate the properties of the circumstellar environment, we modeled the SED with the 1D code DUSTY (Ivezic et al. 1999). For this analysis, we used the photometric data from Miroshnichenko et al. (2005), who discussed the variability of the source. From their Fig. 1, it can be deduced that both $V$ and $K$ magnitudes vary $6-7 \%$ over a $\sim 17$-year time. We assumed average values for $V$ and $K$ magnitudes from the data shown by Miroshnichenko et al. (2005) and derived the other magnitudes accordingly from the average color indices given in the same work. These data were complemented by our new IRAC and IRS observations, as well as by survey measurements from the InfraRed Astronomical Satellite (Neugebauer et al. 1984, IRAS), WISE, and AKARI (Murakami et al. 2007), as shown in Fig. 8.

The figure displays a shallow energy distribution at nearIR wavelengths. Miroshnichenko et al. (2005) had already noticed an excess of emission in the near-IR, which they attributed to a compact gaseous nebula around the central star. This can be identified as the same compact nebula that is generating our IRS emission lines and that was detected by Gauba et al. (2003).

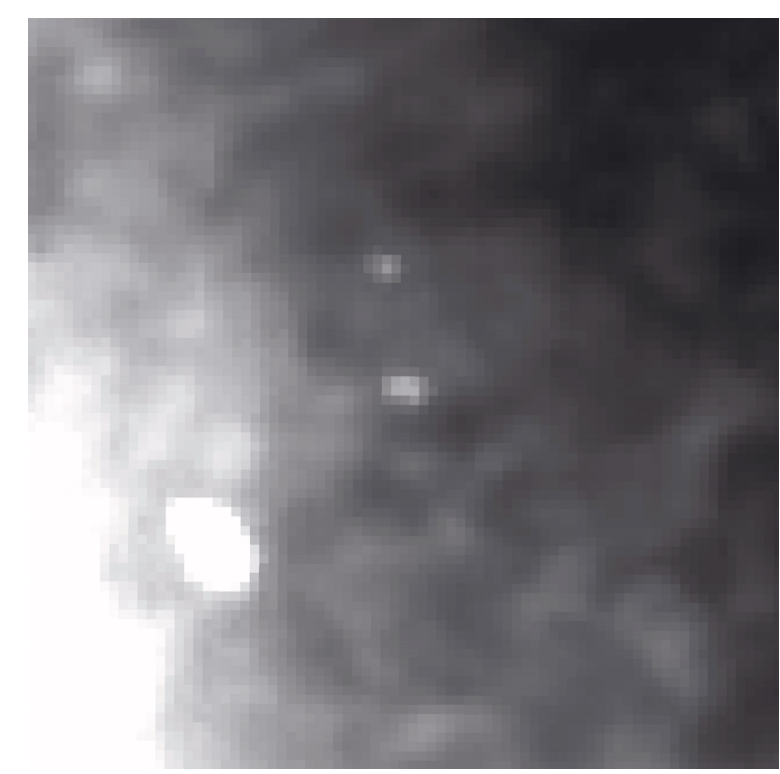

Fig. 7. Cut-out of the IRAS atlas around MWC 930 at $60 \mu \mathrm{m}\left(2^{\circ} \times 2^{\circ}\right)$. North is up and east to the left.

The optical and near-IR observations were dereddened before performing the modeling according to Cardelli et al. (1989). In doing so, we took into account the values of extinction given by Miroshnichenko et al. (2005), who give an interstellar color excess of about 2.5 .

The modeling was performed by assuming an MRN distribution of the radii of the spherical dust grains (Mathis et al. 1977) with a minimum radius of $0.005 \mu \mathrm{m}$, a maximum of $0.25 \mu \mathrm{m}$, and a power law index of -3.5 . The optical constants were those for astronomical silicates given by Draine \& Lee (1984).

\subsection{From UV to mid-IR}

As the source of energy, we took a combination of two Planck curves to reproduce the central star and the compact circumstellar gas. Even such a configuration would not allow us to reach a good fit of the infrared data, as excess emission would still be present at IRAC wavelengths. Therefore, we decided to add 


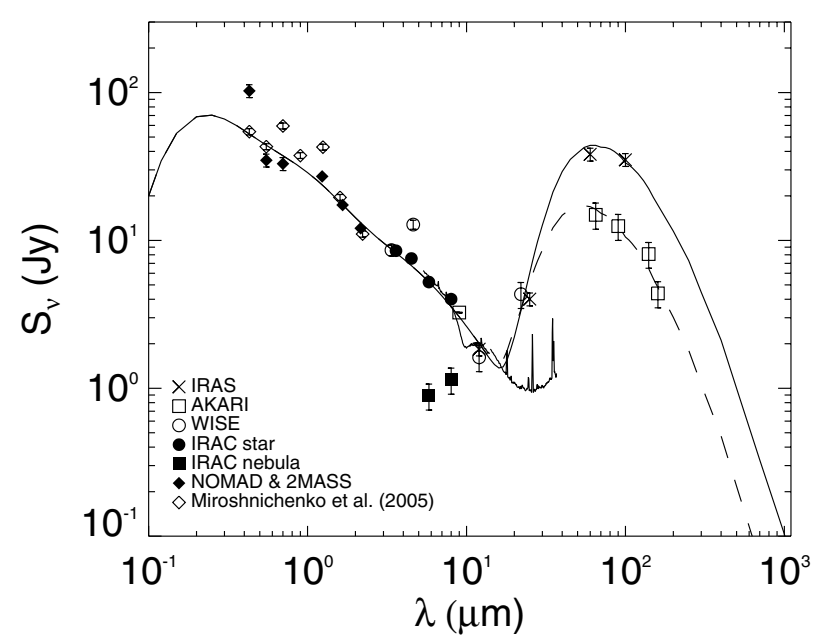

Fig. 8. SED of MWC 930 with overlaid DUSTY outputs: a solid line for the model that reproduces the IRAS far-IR data and a dashed line for the model that reproduces the AKARI points.

a third Planck curve and adjusted the relative luminosities and temperatures of the three central sources to best fit the data, keeping the temperature of the central star set to $22000 \mathrm{~K}$.

The outcome of the DUSTY modeling is displayed in Fig. 8. The temperatures of the central Planck curves are 22000 , 5000 , and $1100 \mathrm{~K}$ with contributions to the total luminosity at $95.5 \%, 4.2 \%$ and $0.3 \%$, respectively. While the extra contribution at $5000 \mathrm{~K}$ can be circumstellar gas or even a companion star, as proposed by Miroshnichenko et al. (2005), the colder component at $1100 \mathrm{~K}$ may be interpreted as due to another gaseous component (an older eruption, for example) or it may point to the existence of hot dust around the star. For a distance of $3.5 \mathrm{kpc}$, Miroshnichenko et al. (2005) determines a total luminosity of about $10^{5.6} L_{\odot}$. After dereddening, we find that a value of $10^{5.7} L_{\odot}$ is more adequate to model the data.

\subsection{The far-IR}

With the far-IR shape of the SED shown in Fig. 8, it is easy to recognize that any gray body fitting the far-IR points (either the AKARI or IRAS data) does not fit the two IRAC measurements of the nebular emission and falls well below them. The color temperature derived from the two IRAC measurements is $T_{\mathrm{c}}=360 \pm 70 \mathrm{~K}$, a value much larger than what is expected for the shell generating the far-IR peak. Observations at other wavelengths are desirable to better sample the SED of the nebula that are detected with IRAC. If the two points are really due to warm dust, it is not clear how this would be kept at high temperature so far away from the central star.

An alternate possibility is that the IRAC photometric measurements are strongly contaminated by line emission. Around 5.8 and $8.0 \mu \mathrm{m}$, the IRS spectrum is dominated by the radiation from the central source and the examination of the BCDs after background subtraction did not show hints for emission from the detached shell. We are therefore unable to say whether the IRAC fluxes are contaminated by lines.

The AKARI and IRAS observational points clearly disagree. Such a discrepancy may be due to the IRAS photometric measurement being performed over the whole source, while the AKARI one, which has a smaller PSF, is missing extended emission. The FWHM of the PSF of the AKARI Far-Infrared Surveyor (FIS) detectors are 37" (at $65 \mu \mathrm{m}), 39^{\prime \prime}(90 \mu \mathrm{m})$, $58^{\prime \prime}(140 \mu \mathrm{m})$ and $61^{\prime \prime}(160 \mu \mathrm{m})$, which must be compared to a nebular diameter of about $100^{\prime \prime}$. It is therefore possible that the PSF-fitting photometry of the AKARI catalog underestimates the emission of the extended nebula. Unfortunately, AKARI images are not yet publicly available to allow us to perform the photometry of our target. Furthermore, the values at 65, 140, and $160 \mu \mathrm{m}$ are listed in the catalog as "low reliability", as the source is not confirmed.

In principle, it might also be true that the IRAS measurements overestimate the emission from the source because of contamination from nearby material. We showed in Fig. 7 that the IRAS images display a field rich in diffuse emission.

In the attempt to figure out whether the IRAS or the AKARI data set is more reliable, we modeled the SED twice, once matching the IRAS points and once matching the AKARI ones. When fitting the IRAS data points, we obtain a dust temperature of the nebula at its inner radius $\left(R_{\text {in }}\right)$ of $63 \mathrm{~K}$ and an opacity in $V$ of $1.1 \times 10^{-3}$. From the output of DUSTY, we can estimate $R_{\text {in }} \sim 3.2 \times 10^{18} \mathrm{~cm}(\sim 1.04 \mathrm{pc})$, which at the assumed distance equals $\sim 63^{\prime \prime}$. If we fit the AKARI data, instead of the IRAS ones, the dust temperature at $R_{\text {in }}$ is then $75 \mathrm{~K}$, the opacity at $0.55 \mu \mathrm{m}$ is $5 \times 10^{-4}$, and $R_{\text {in }} \sim 1.9 \times 10^{18} \mathrm{~cm}(\sim 0.62 \mathrm{pc})$, which gives about $38^{\prime \prime}$. The dust masses of the nebula also differ in the two cases. By matching the IRAS data, we obtain a dust mass of $0.12 M_{\odot}$. With the AKARI points, we have $0.02 M_{\odot}$. Neither of the two fits returns an inner radius very close to that observed with IRAC $\left(\sim 46^{\prime \prime}\right)$.

Assuming a standard gas-to-dust mass ratio of 100, the values of dust mass would correspond to 12 and $2 M_{\odot}$ for the IRAS and AKARI data fit, respectively. A substantial amount of mass was then lost when the nebula was ejected, especially if we consider that MWC 930 is not a very luminous LBV. Miroshnichenko et al. (2005) estimated that its mass on the main sequence was probably around $35 M_{\odot}$. Both values of dust mass are large for a single eruptive event. It should be considered that the calculation of the mass from the model output depends on the dust opacity at sub-mm wavelengths; therefore, if the grain properties are not correct, then neither is the derived mass. For example, it is known that in AGB and post-AGB stars, the submm dust emissivity corresponds with $\lambda^{-1}$ rather than $\lambda^{-1.7}$, as it is observed in the interstellar medium and assumed in standard sets of optical constants for astronomical dust (Justtanont \& Tielens 1992; Gürtler et al. 1996). This shallower slope may be due to a different chemical composition of the grains or a different size distribution, since larger grains emit more efficiently at large wavelengths. A similar difference for LBVs, where dust grains are relatively "fresh", such as in AGB stars, would lower our mass estimates of about an order of magnitude.

\subsection{The silicate feature at $9.7 \mu \mathrm{m}$}

In Sect. 4, we noticed that it is not clear whether the silicate absorption feature in the IRS spectrum is of circumstellar or interstellar origin. To figure out which is the case, we now take advantage of the plots in Fig. 9 obtained with DUSTY for the values of opacity at $0.55 \mu \mathrm{m}$ and dust temperature at the inner radius indicated in the figure. The models were calculated taking a Planck curve at $2 \times 10^{4} \mathrm{~K}$ as the central source, astronomical silicates from Draine \& Lee (1984) to describe the grain properties, and a ratio of outer to inner dust radius of 200.

As we can see, the feature is observed in absorption only at large values of opacity for both hot and cold dust. For small $(<1)$ opacity values, it is only seen in emission when the dust is hot, or it is not seen at all when the dust is cold. 

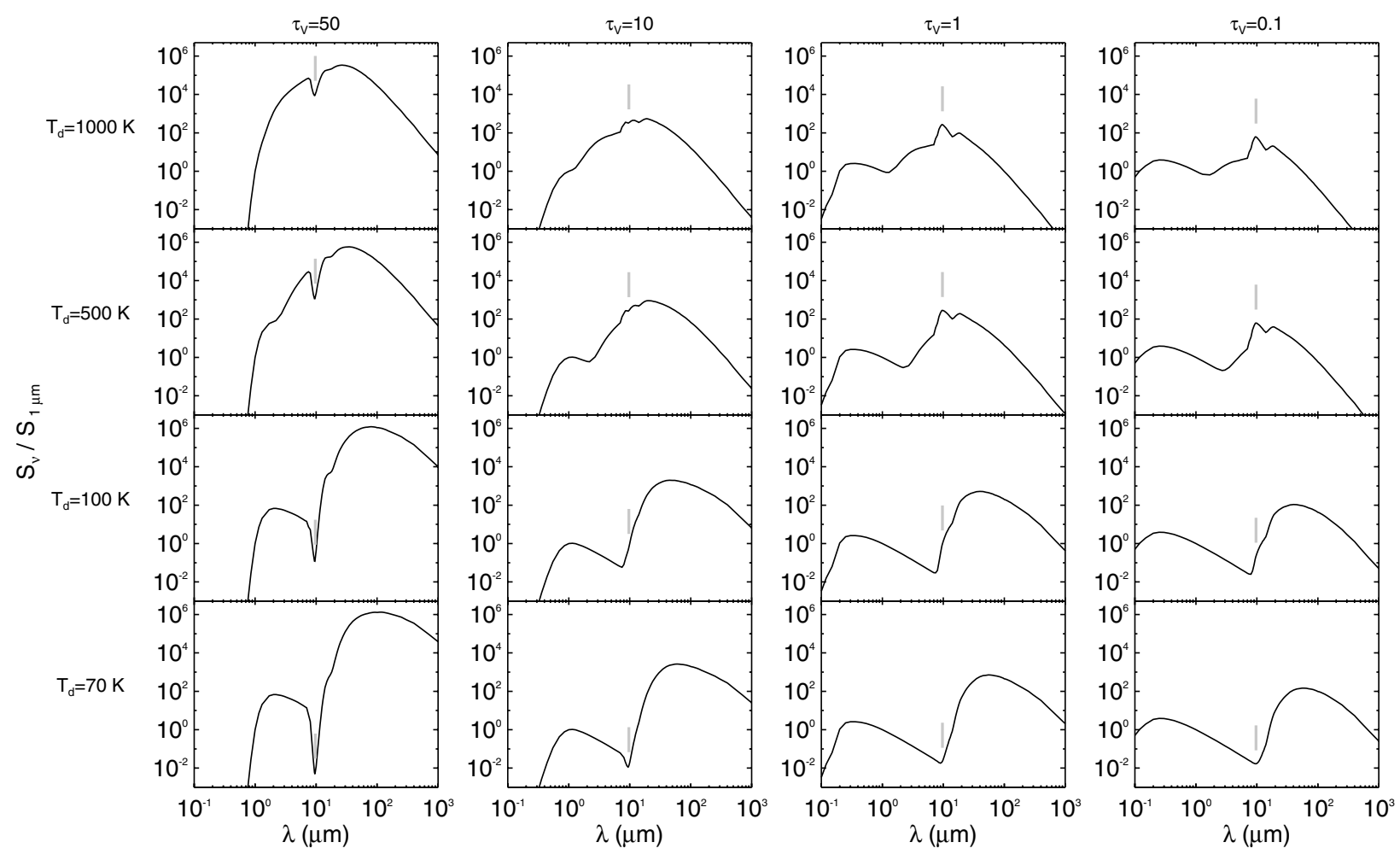

Fig. 9. DUSTY models obtained at various values of dust opacity and temperature. A gray bar indicates the wavelength of $9.7 \mu \mathrm{m}$ in every plot.

In our source, the feature could be due either to the extended nebula seen with IRAC or to the compact and hot shell at a few arcsec from the star. In either case, the opacity of the dust should be larger than 1 for the feature to be observed in absorption over a hot continuum.

A B-type star, like the central source in MWC 930, emits most of its energy at UV and optical wavelengths, where dust absorption is most efficient. For high opacity values $\left(\tau_{V}>1\right)$, the absorption of the large amount of energy from the central star would determine an intense emission in the far-IR. One would then expect the far-IR peak to be much brighter than the optical/UV peak. This scenario does not match the observational data, where the far-IR peak is only slightly brighter than the optical one before dereddening and is actually less bright than the latter after dereddening. Therefore, the circumstellar opacity in $V$ is unlikely to be larger than 1 , which leads us to conclude that the absorption feature is of interstellar origin.

\section{Summary and conclusions}

We have detected a large $\left(100^{\prime \prime} \times 85^{\prime \prime}\right)$ circumstellar nebula around the LBV candidate MWC 930 at mid-IR wavelengths, which strengthens the classification of the source as an LBV.

As seen at other wavelengths, the mid-IR spectrum is characterized by lines of [FeII] and a continuum displaying infrared excess emission, if compared to that of a star with $T_{\mathrm{eff}}=22000 \mathrm{~K}$. Some lines cannot be clearly attributed because of the low spectral resolution of our data, but the examination of the spectrum indicates that the lines are produced in a compact region of a few arcsec around the star, whose existence had been noticed before by Miroshnichenko et al. (2005) and Gauba et al. (2003). The comparison of the intensities of the [FeII] and [SiII] lines with model PDR values implies that this compact shell has a high density of at least $10^{4} \mathrm{~cm}^{-3}$.
By 1D modeling of the SED, we conclude that two more components must be taken into account as central sources beside the central star, with one at $5000 \mathrm{~K}$ and the other at $1100 \mathrm{~K}$. While the former is certainly gaseous, the latter may actually be hot dust condensing close to the star, which would imply that dust formation is going on in the circumstellar environment. The outer nebula detected with IRAC is much colder $(63 \mathrm{~K}$ at its inner radius) and seems to contain a substantial amount of the original stellar mass.

Acknowledgements. L.C. acknowledges funding from the Spanish Consejo Superior de Investigaciones Cientificas through a JAE-Doc research contract co-funded by the European Social Fund. L.C. thanks the Spanish MICINN for funding support through grants AYA2009-07304 and CSD2009-00038. This work is based in part on observations made with the Spitzer Space Telescope, which is operated by the Jet Propulsion Laboratory, California Institute of Technology, under a contract with NASA. Support for this work was provided by NASA through an award issued by JPL/Caltech. This publication makes use of data products from the Wide-field Infrared Survey Explorer, which is a joint project of the University of California, Los Angeles, and the Jet Propulsion Laboratory/California Institute of Technology, funded by the National Aeronautics and Space Administration. This research is based on observations with AKARI, a JAXA project with the participation of ESA.

\section{References}

Cardelli, J. A., Clayton, G. C., \& Mathis, J. S. 1989, ApJ, 345, 245

Carmona, A., van den Ancker, M. E., Audard, M., et al. 2010, A\&A, 517, A67 Chiar, J. E., \& Tielens, A. G. G. M., ApJ, 637, 774

Draine, B. T. 1978, ApJS, 36, 595

Draine, B. T. \& Lee, H. M. 1984, ApJ, 285, 89

Fazio, G., Hora, J. L., Allen, L. E., et al. 2004, ApJS, 154, 10

Gauba, G., Parthasarathy, M., Kumar, B., Yadav, R. K. S., \& Sagar, R. 2003, A\&A, 404, 305

Gürtler, J., Kömpe, C., \& Henning, Th. 1996, A\&A, 305, 878

Habing, H. J. 1968, Bull. Astr. Inst. Netherlands, 19, 421

Higdon, S. J. U., Devost, D., Higdon, J. L., et al. 2004, PASP, 116, 975

Houck, J., Roellig, T. L., van Cleve, J., et al. 2004, ApJS, 154, 18

Humphreys, R. M. \& Davidson, K. 1994, PASP, 106, 1025 
Ishihara, D., Onaka, T., Kataza, H., et al 2010, A\&A, 514, A1

Ivezic, Z., Nenkova, M., \& Elitzur, M. 1999, User Manual for DUSTY, University of Kentucky Internal Report, available at http://www.pa.uky. edu/ moshe/dusty

Justtanont, K., \& Tielens, A. G. G. M., 1992, ApJ, 389, 400

Kaufman, M. J., Wolfire, M. G., Hollenbach, D. J., \& Luhman, M. L. 1999, ApJ, 527,795

Kaufman, M. J., Wolfire, M. G., \& Hollenbach, D. J. 2006, ApJ, 644, 283

Makovoz, D., Roby, T., Khan, I., \& Booth, H. 2006, Proc. SPIE, 6274, 0C

Marengo, M., Megeath, T., Fazio, G., et al. 2006, ASP Conf. Ser., 357, 105

Marengo, M., Hora, J. L., Barmby, P., et al. 2007, ASP Conf. Ser., 378, 80

Mathis J. S., Rumpl W., \& Nordsieck K. H. 1977, ApJ, 217, 425

Miroshnichenko, A. S. Bjorkman, K. S., Grosso, M., et al. 2005, MNRAS, 364, 335
Molster, F. J., Waters, L. B. F. M., \& Kemper, F. 2010, Lect. Notes Phys., 815, 143

Murakami, H., Baba, H., Barthel, P., et al. 2007, PASJ, 59, 369

Neugebauer, G., Habing, H. J., van Duinen, R., et al. 1984, ApJ, 278, L1

Nota, A., Livio, M., Clampin, M., \& Schulte-Ladbeck, R. 1995, ApJ, 448, 788

Parthasarathy, M., Vijapurkar, J., \& Drilling, J. S. 2000, A\&AS, 145, 269

Schuster, M., Marengo, M., \& Patten, B. M. 2006, Proc. SPIE, 6270, 20

Umana, G., Buemi, C. S., trigilio, C., et al. 2009, ApJ, 694, 697

Umana, G. Buemi, C. S., Trigilio, C., Leto, P., \& Hora, J. L. 2010, ApJ, 718, 1036

Venkata Raman, V., \& Anandarao, B. G. 2008, MNRAS, 385, 1076

Vink, J. S. 2012, Astrophys. Space Sci. Lib., 384, 221

Werner, M. W., Roellig, T. L., Low, F. J., et al. 2004, ApJS, 154, 1

Wright, E. L., Eisenhardt, P. R. M., Mainzer, A. K., et al. 2010, AJ, 140, 1868 